\title{
Argon retentivity of carbonaceous materials: feasibility of kerogen as a carrier phase of Q-noble gases in primitive meteorites
}

\author{
Takahito Osawa ${ }^{1}$, Norie Hirao $^{2}$, Nobuyori Takeda $^{3}$, and Yuji Baba ${ }^{2}$ \\ ${ }^{1}$ Neutron Imaging and Activation Analysis Group, Quantum Beam Science Directorate, Japan Atomic Energy Agency (JAEA), \\ Tokai-mura, Naka-Gun, Ibaraki, 319-1195, Japan \\ ${ }^{2}$ Surface Chemistry Research Group, Synchrotron Radiation Research Unit, Quantum Beam Science Directorate, \\ Japan Atomic Energy Agency (JAEA), Tokai-mura, Naka-Gun, Ibaraki, 319-1195, Japan \\ ${ }^{3}$ JAPEX Research Center, Japan Petroleum Exploration Co. Ltd., Hamada 1-2-1, Mihama-ku, Chiba, 261-0025, Japan
}

(Received January 9, 2009; Revised March 19, 2009; Accepted May 27, 2009; Online published October 19, 2009)

\begin{abstract}
Extremely large amounts of heavy noble gases are concentrated in phase Q, which seems to be a carbonaceous phase analogous to terrestrial Type III kerogen. Phase Q must have very high noble gas retentivity based on the presence of such extremely large amounts of heavy noble gases in a very minor fraction of the meteorite. To verify that kerogen is a carrier phase of Q-noble gases, X-ray absorption spectroscopy (XAS) and X-ray photoelectron spectroscopy (XPS) using synchrotron radiation were carried on for kerogens (coals) and carbon allotropes that had been bombarded by $3-\mathrm{keV}$ Ar ions, and the Ar retentivities of the two materials were compared. This comparison of the estimated Ar concentrations in the target materials revealed that carbon allotropes (graphite, fullerene, carbon nanotube, and diamond) have a much higher Ar retentivity than kerogens. This unexpected result clearly shows that the terrestrial kerogens tested in our study are not suitable as a carrier phase of Ar and, consequently, that phase Q may not be similar to the terrestrial kerogen tested. If heavy noble gases are really concentrated in carbonaceous components of primitive meteorites, phase Q may have a more ordered structure than terrestrial kerogen based on the fact that the greatest difference between terrestrial kerogen and carbon allotropes is the degree of order of the molecular structure.
\end{abstract}

Key words: Noble gas, XAS, XPS, kerogen, phase Q, ion implantation, diamond, graphite.

\section{Introduction}

Primordial trapped components of noble gases preserved in meteorites provide important information on the early solar system. A large number of trapped noble gas components are preserved in distinctive phases of meteorites. Of these, Q-noble gases are the most important component because heavy noble gases in primitive chondrites are mainly hosted by phase $\mathrm{Q}$, which is an oxidizable phase of a residue of treatment with hydrochloric acid and hydrofluoric acid (e.g., Lewis et al., 1975; Ott et al., 1981; Huss et al., 1996). Phase Q is also the carrier of about $15 \%$ of the $\mathrm{He}$ and Ne. Phase Q is operationally defined as the major carrier of the heavy noble gases normally trapped in Allende, which releases noble gases upon treatment with oxidizing acids (Lewis et al., 1975). This latter process indicates that the phase is easily oxidized. Although the nature of the residual material after $\mathrm{HCl} / \mathrm{HF}$ treatment is ill identified, phase Q seems to be a carbonaceous phase (Ott et al., 1981), and insoluble organic matter in the meteorite is analogous to terrestrial Type III kerogen, which is a mixture of organic material formed from terrestrial plant matter (Hayatsu et al., 1983; Murae, 1994; Gardinier et al., 2000; Remusat et al., 2003). Heavy noble gases in the insoluble organic matter of a primitive chondrite are largely lost upon pyridine treat-

Copyright (c) The Society of Geomagnetism and Earth, Planetary and Space Sciences (SGEPSS); The Seismological Society of Japan; The Volcanological Society of Japan; The Geodetic Society of Japan; The Japanese Society for Planetary Sciences; TERRAPUB. ment, suggesting that there are at least two substructures in phase Q and that Q-noble gases may be trapped in organic layers (Marrocchi et al., 2005a). Their results also show that Q-noble gases are not captured in the inorganic matters.

Noble gases preserved in phase $\mathrm{Q}$ have several remarkable properties. One of the most notable characteristics is that noble gases, particularly heavy noble gases ( $\mathrm{Ar}, \mathrm{Kr}$ and $\mathrm{Xe})$, are extremely enriched in a very small fraction. According to one model, $0.04 \mathrm{wt} \%$ of the meteorite bulk is phase Q (Anders, 1981), but this value is only an upper limit. More than $90 \%$ of total Xe virtually exists in the phase Q of CI chondrite (Huss and Lewis, 1995). Noble gases are strongly retained in this phase and have a high release temperature of over $1000^{\circ} \mathrm{C}$ even under ultra-high vacuum (e.g., Huss et al., 1996; Busemann et al., 2000). This fact means that phase $Q$ has a very high retentivity of noble gases. The encapsulating mechanism of noble gases is, however, still unknown. Although several mechanisms are suggested, such as active capture (Hohenberg et al., 2002), condensation (Frick, 1979), diffusion (Zadnik et al., 1985), ion implantation (Matsuda and Yoshida, 2001), and low pressure adsorption (Wacker et al., 1985; Wacker, 1989; Marrocchi et al., 2005b), these processes cannot completely reproduce the composition and high concentration of Q-noble gases. Although low-pressure adsorption is a plausible model to reproduce the composition of Q-noble gas, in this model Type III kerogen cannot adequately ac- 
count for the noble gas inventory due to its low adsorption capacity (Marrocchi et al., 2005b).

Another remarkable nature of phase $\mathrm{Q}$ is its ordinariness. The general presence of Q-noble gases in different types of primitive stony meteorites (e.g., Wieler et al., 1991; Busemann and Eugster, 2002) indicates that a common process which occurred in the asteroid region of the early solar nebula tightly fixed heavy noble gases to phase Q-forming materials. Ozima et al. (1998) reported that the elemental and isotopic compositions of Q-noble gases show some similarity with those of solar noble gases, suggesting the possibility that Q-noble gases may have been formed through the capture of these gases in the early solar nebula.

The carrier phase of Q-noble gases must have a high retentivity of noble gases whatever the trapping mechanism involved. If the carrier phase is truly carbonaceous material, noble gases should be encapsulated in the carbon network of molecules or the medium between layers of aromatic moieties. In this study, X-ray photoelectron spectroscopy (XPS) spectra and X-ray absorption spectra (XAS) of carbon allotropes and kerogens (coals) irradiated by a 3$\mathrm{keV} \mathrm{Ar}$ ion beam were recorded in order to evaluate their retentivity and trapping mode of Ar. Argon is the secondmost abundant element in Q-noble gases after He. Although phase $\mathrm{Q}$ is identified in meteorites based on the presence and abundance of $\mathrm{Xe}$, the abundance ratio of ${ }^{36} \mathrm{Ar} /{ }^{132} \mathrm{Xe}$ is $76 \pm 7$ (Busemann et al., 2000). We adopted the 3-keV ion bombardment as a noble gas implantation mechanism in our study because only retentivity was evaluated. This mechanism can inject excessive amounts of Ar into the target material, and the concentration of $\mathrm{Ar}$ in the target material strongly depends on the escape rate of Ar from the target rather than the amount of bombarded Ar. The ion implantation partially destroys and sputters the target material and, consequently, Ar concentration depends on the incident energy of the injected ions and the composition of the target material. However, carbonaceous materials were tested in the study reported here, and the sputtering yield of carbon was found to be much smaller than that of other major elements (Greene et al., 1997). It is known that highenergy (30 keV or more) ion implantation can efficiently produce doped fullerene (Kastner et al., 1993; Watanabe et al., 2003a, b). Fan et al. (1994) reported that Ar-doped $\mathrm{C}_{60}$ was produced by the $80-\mathrm{keV} \mathrm{Ar}^{+}$implantation. Since there was no obvious difference in infrared spectrum between unimplanted and implanted $\mathrm{C}_{60}$, these researchers concluded that the $80-\mathrm{keV}$ ion bombardment did not notably destroy the cage structure of fullerene. Since the energy of the ion $(3 \mathrm{keV})$ bombardment we adopted is much lower than that used in previous experiments, the degree of destruction of the target materials is lower. As such, the ion bombardment is a suitable method for evaluating the retentivity of Ar, and the escape rate does not depend on the noble gas implantation mechanism but only on the diffusion coefficient of the noble gas in the material.

\section{Samples}

Four carbon allotropes were prepared: Highly Oriented Pyrolytic Graphite (HOPG) (Alfa Aesar), carbon nanotube (multi-walled, 20-30 nm), fullerene mixture (nanom mix
$\mathrm{ST}, \mathrm{C}_{60} 60 \%, \mathrm{C}_{70} 25 \%$, higher-order fullerene $15 \%$ ), and Chemical Vapor Deposition (CVD) diamond (Sumitomo Electric). High-purity iron and San Carlos olivine were also prepared for reference. In order to obtain a clean target surface, we initially sputtered the samples with $3-\mathrm{keV} \mathrm{Ar}^{+}$ ions for $1 \mathrm{~min}$ at an angle of $45^{\circ}$ from the surface normal.

The seven coal samples were collected in Hokkaido, north Japan in 1975. Coal represents terrestrial kerogen, which is a decay product of biological materials. Kerogen is insoluble in organic solvents (pyridine and carbon disulfide) due to its large molecular structure, and the soluble portion is known as bitumen. Although kerogen is a mixture of organic compounds and the structure is extremely complex, it can be classified into three main types based on a chemical composition. The chemical compositions of the seven coal samples were investigated using three analytical methods: elemental (CHN) analysis using the J-Science Laboratory's JM-10 Micro Corder (CHN corder); Rock-Eval pyrolysis method by VINCI Technologies Rock-Eval 6 S3 Classic; energy-dispersive X-ray spectroscopy (EDS) using a JEOL JED-2200F. In the CHN analysis, a powdered coal sample was first treated with $\mathrm{HCl}$ and the carbonate removed. A 2- to 3-mg sample of the residue was heated at $950^{\circ} \mathrm{C}$ in a $\mathrm{He} / \mathrm{O}_{2}$ mixing gas and the released $\mathrm{H}_{2} \mathrm{O}, \mathrm{CO}_{2}$, and $\mathrm{N}_{2}$ gases measured. This analysis yielded information on the concentration of $\mathrm{C}, \mathrm{H}$, and N. The Rock-Eval pyrolysis method (Espitalié et al., 1980) is now widely used for the standard characterization of organic matter without kerogen isolation. In programmed-temperature pyrolysis, about $10 \mathrm{mg}$ of a powdered sample, which was not treated by acid, is gradually heated in $\mathrm{N}_{2}$ gas at $300-600^{\circ} \mathrm{C}$, and the released hydrocarbons and $\mathrm{CO}_{2}$ subsequently measured. These analyses can determine the major elemental composition of coals. The third method, EDS, was also used to investigate the composition of the heavier (minor) elements in the coals. The results of CHN analysis and Rock-Eval pyrolysis are summarized in Tables 1 and 2, respectively. In Table 2, $S_{1}$, $\mathrm{S}_{2}$, and $\mathrm{S}_{3}$ are the amounts of free hydrocarbons, hydrocarbons generated through thermal cracking of kerogen, and $\mathrm{CO}_{2}$ produced during pyrolysis of kerogen, respectively. $\mathrm{S}_{2}$ is mainly released at $400-440^{\circ} \mathrm{C}$. The hydrogen index (HI) and oxygen index (OI), which correlate with the $\mathrm{H} / \mathrm{C}$ and $\mathrm{O} / \mathrm{C}$ atomic ratios, respectively, can be used to characterize the origin of the organic matter. The production index (PI = $\left.\mathrm{S}_{1} /\left(\mathrm{S}_{1}+\mathrm{S}_{2}\right)\right)$ is used to determine the maturity of kerogen and to detect the generation of hydrocarbons. Generally, the PI increases with subsurface depth and organic maturity.

Kerogens are classified into three main types called Types I, II, and III (Durand and Monin, 1980). Type I is quite uncommon because it is derived mainly from lacustrine algae. Figure 1 is a HI/OI diagram (Rock-Eval van Krevelen-type diagram, van Krevelen, 1950) showing that the coals are widely distributed in the range between Type II and III kerogen. They are not classified into Type I kerogen due to their low HI. Some coals had detectable amounts of $\mathrm{Mg}, \mathrm{Al}, \mathrm{Si}, \mathrm{S}, \mathrm{K}, \mathrm{Ca}$, and $\mathrm{Fe}$, as measured by EDS, and these EDS results were used to calculate the total photon cross section of the coals. 
Table 1. Chemical compositions of coals.

\begin{tabular}{|c|c|c|c|c|c|c|c|c|c|}
\hline \multirow{2}{*}{ Coal sample } & \multirow{2}{*}{$\begin{array}{c}\text { Carbonate } \\
(\text { wt } \%)^{\mathrm{a}}\end{array}$} & \multicolumn{3}{|c|}{ residue of $\mathrm{HCl}$ treatment $(\mathrm{wt} \%)$} & \multicolumn{3}{|c|}{ whole rock $(\text { wt } \%)^{\mathrm{b}}$} & \multirow{2}{*}{$\mathrm{H} / \mathrm{C}$} & \multirow{2}{*}{$\mathrm{N} / \mathrm{C}$} \\
\hline & & $\mathrm{C}$ & $\mathrm{H}$ & $\mathrm{N}$ & $\mathrm{C}$ & $\mathrm{H}$ & $\mathrm{N}$ & & \\
\hline Shin-sarufutsu & 2.41 & 56.0 & 5.1 & 1.3 & 54.7 & 5.0 & 1.3 & 1.09 & 0.02 \\
\hline Mitsui Ashibetsu & 5.12 & 76.8 & 5.5 & 2.2 & 72.9 & 5.2 & 2.0 & 0.86 & 0.02 \\
\hline Mitsui Bibai & 2.13 & 70.3 & 5.8 & 1.5 & 68.8 & 5.7 & 1.5 & 0.99 & 0.02 \\
\hline Mitsui Bibai (py) $^{\mathrm{c}}$ & 1.04 & 65.4 & 5.0 & 1.7 & 64.7 & 4.9 & 1.7 & 0.91 & 0.02 \\
\hline Tomiuchi Fukuyama & 5.97 & 59.6 & 4.7 & 1.6 & 56.1 & 4.4 & 1.5 & 0.95 & 0.02 \\
\hline${\text { Tomiuchi Fukuyama }(p y)^{c}}^{c}$ & 2.20 & 59.1 & 4.4 & 1.9 & 57.8 & 4.3 & 1.8 & 0.90 & 0.03 \\
\hline Nukibetsu & 4.36 & 30.8 & 2.6 & 0.6 & 29.4 & 2.5 & 0.6 & 1.01 & 0.02 \\
\hline Nukibetsu (py) ${ }^{\mathrm{c}}$ & 4.59 & 17.7 & 1.4 & 0.7 & 16.8 & 1.3 & 0.6 & 0.93 & 0.03 \\
\hline Obihiro & 10.71 & 53.8 & 4.8 & 0.9 & 48.1 & 4.2 & 0.8 & 1.06 & 0.01 \\
\hline Taiheiyo Kushiro & 4.38 & 66.9 & 5.3 & 1.2 & 63.9 & 5.1 & 1.2 & 0.96 & 0.02 \\
\hline Taiheiyo Kushiro (py) ${ }^{\mathrm{c}}$ & 1.00 & 60.9 & 4.4 & 1.5 & 60.3 & 4.4 & 1.4 & 0.87 & 0.02 \\
\hline
\end{tabular}

Table 2. Rock-eval pyrolysis results for coals.

\begin{tabular}{lcccccccc}
\hline \multicolumn{1}{c}{ Coal sample } & $\mathrm{T}_{\max }\left({ }^{\circ} \mathrm{C}\right)^{\mathrm{a}}$ & $\mathrm{S}_{1}(\mathrm{mg} / \mathrm{g})$ & $\mathrm{S}_{2}(\mathrm{mg} / \mathrm{g})$ & $\mathrm{S}_{3}(\mathrm{mg} / \mathrm{g})$ & $\mathrm{TOC}(\mathrm{wt} \%)^{\mathrm{b}}$ & $\mathrm{HI}^{\mathrm{c}}$ & $\mathrm{OI}^{\mathrm{d}}$ & $\mathrm{PI}^{\mathrm{e}}$ \\
\hline Shin-sarufutsu & 423 & 4.6 & 195.1 & 9.3 & 54.7 & 357 & 17 & 0.02 \\
Mitsui Ashibetsu & 433 & 12.3 & 193.0 & 7.0 & 72.9 & 265 & 10 & 0.06 \\
Mitsui Bibai & 415 & 3.8 & 106.1 & 20.6 & 68.8 & 154 & 30 & 0.03 \\
Tomiuchi Fukuyama & 415 & 3.8 & 106.1 & 20.6 & 56.1 & 189 & 37 & 0.03 \\
Nukibetsu & 422 & 0.9 & 21.9 & 18.7 & 29.4 & 74 & 64 & 0.04 \\
Obihiro & 405 & 8.0 & 77.5 & 54.7 & 48.1 & 161 & 114 & 0.09 \\
Taiheiyo Kushiro & 416 & 4.3 & 166.4 & 16.5 & 63.9 & 260 & 26 & 0.03 \\
\hline
\end{tabular}

${ }^{\mathrm{a}}$ The temperature at which the maximum release of hydrocarbons

${ }^{\mathrm{b}}$ Total Organic Carbon

${ }^{\mathrm{c}}$ Hydrogen index, which is defined as $\mathrm{S}_{2} / \mathrm{TOC} \times 100$

a Oxygen index, which is defined as $\mathrm{S}_{3} / \mathrm{TOC} \times 100$

${ }^{\mathrm{e}}$ Production index, which is defined as $\mathrm{S}_{1} /\left(\mathrm{S}_{1}+\mathrm{S}_{2}\right)$

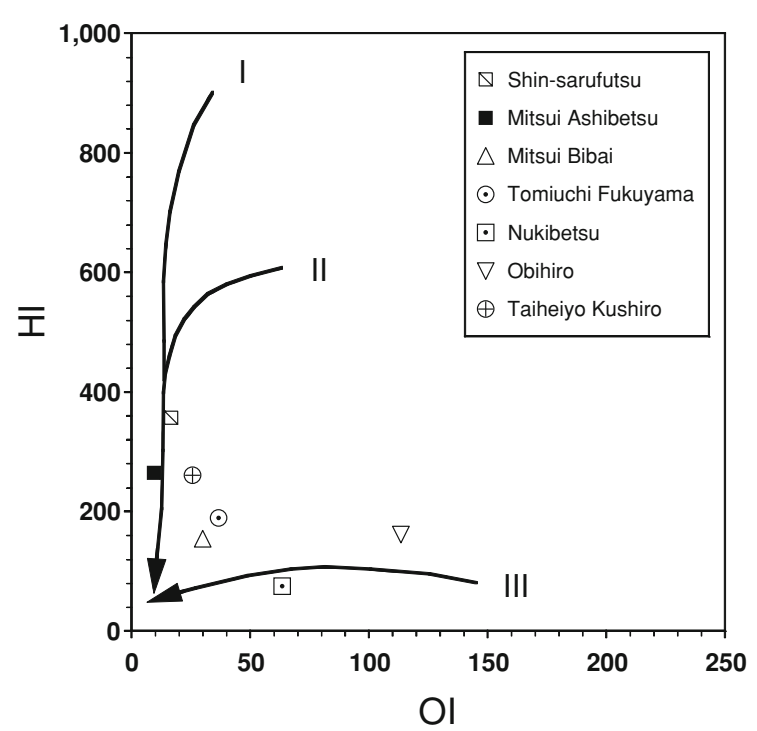

Fig. 1. Hydrogen index (HI)/oxygen index (OI) plot for Hokkaido coals from Rock Eval pyrolysis data. HI and OI are defined as $\left[\mathrm{S}_{2} / \mathrm{TOC}\right] \times 100$ and $\left[\mathrm{S}_{3} / \mathrm{TOC}\right] \times 100$, respectively. $\mathrm{HI}$ is a parameter used to characterize the origin of organic matter (e.g., Hartman-Stroup, 1987). OI is a parameter that correlates with the $\mathrm{O} / \mathrm{C}$ ratio. Three curves indicate the maturation of the organic matter (I: oil prone, II: oil/gas prone, III: gas prone), and the arrows indicate the increasing maturation of kerogen.

\section{Experimental}

XPS is a spectroscopic technique using soft X-ray radiation to study the elemental composition, chemical state, and electronic state of the surface region of a material. This analytical method can quantitatively determine the elemental concentration, but detection limits for most of the elements are higher than 0.1 atom\%. Alternatively, XAS is utilized in various research fields to examine the local structure and electronic state of a sample. Other acronyms, such as XAFS (X-ray absorption fine structure) and XANES (X-ray absorption near-edge structures) are often used.

The XPS and XAS experiments were performed at the BL-27A station of the Photon Factory of the High Energy Accelerator Research Organization (KEK-PF), employing double-crystals of InSb (111) as a monochromator. The analytical systems installed at the station have been described in detail in Hirao et al. (2006). The energy resolution of the monochromator was $E / \Delta E \sim 2000$, or about $1.6 \mathrm{eV}$, where the energy is $3.2 \mathrm{keV}$ ( $\mathrm{Ar} K$-edge). The photon energy was calibrated using the $\mathrm{C} 1 \mathrm{~s}$ photoelectron peak of HOPG (284.3 eV) in XPS. Coals and San Carlos olivine were crushed and powdered in an agate mortar, and the bitumen in some of the coal samples was removed by means of a 1-h ultrasonic extraction with pyridine. The powdered samples (coals, carbon nanotube, fullerene, and olivine) were embedded on the surface of an indium plate and attached to a copper plate by carbon tape in order to reduce the charging effects. Other solid samples were directly set on a cop- 


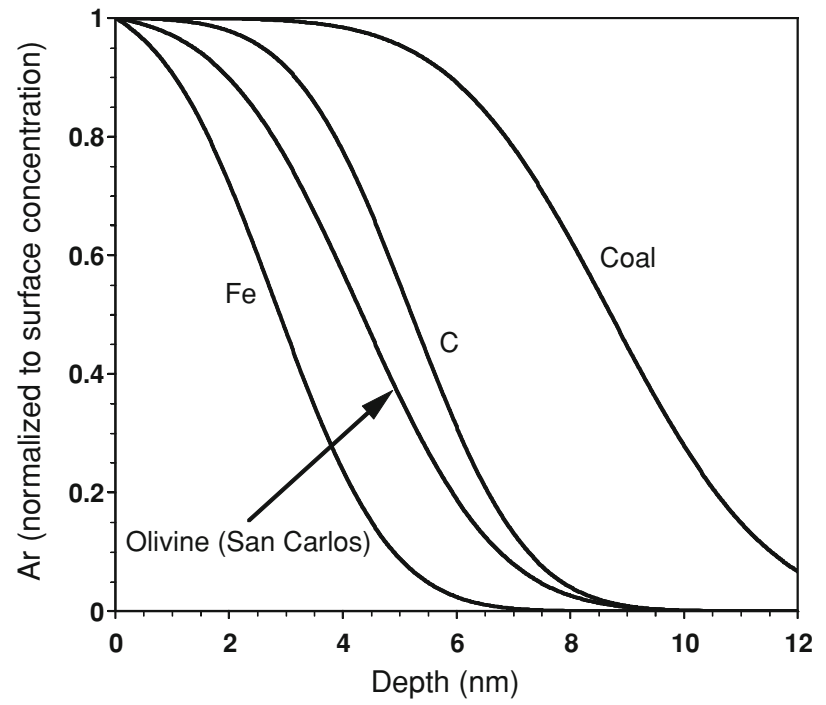

Fig. 2. Depth profiles of quasi-saturated Ar in four targets (C, Fe, San Carlos olivine, and coal) after a long bombardment time. The results of SRIM code simulations are used here, and an arbitrary erosion rate is set in the calculation. The profile of coal is calculated from an average composition of coals tested in this work. The depth profiles are independent of the erosion rate.

per plate. The samples were introduced into an ultra-high vacuum chamber with base pressure of $1 \times 10^{-8} \mathrm{~Pa}$. The analysis chamber consisted of a manipulator, a hemispherical electron energy analyzer (VSW, CLASS-100), and a low-energy electron flood gun. In XPS, the photon beam was $55^{\circ}$ from the surface normal, and the electron takeoff direction was surface normal. XPS was performed at 3.5$\mathrm{keV}$ photon energy to obtain the Ar1s peak. The XAS was obtained from the total electron yields. The spectra were recorded by plotting the sample current as a function of photon energy, with the sample current being normalized to the photon intensity calculated from the drain current of an aluminum foil located in front of the sample.

Argon ions were produced in a cold-cathode ion-gun (OMEGATRON Co. OMI-0045CK), and the target materials were bombarded with $3-\mathrm{keV} \mathrm{Ar}^{+}$ions at room temperature at the surface normal angle for 30-225 min after the initial sputtering. The ion flux was in the range of 2.0$4.5 \mu \mathrm{A} / \mathrm{cm}^{2}$, and the total number of bombarding ions was $2.2-25.4 \times 10^{16}$ ions $/ \mathrm{cm}^{2}$. The bombardments were carried out in the same chamber in which the XPS and XAS analyses were performed. X-ray analyses were started about $10 \mathrm{~min}$ after ion bombardment because the vacuum level was low immediately following the bombardment.

The Ar concentration at the surface region of carbon allotropes could be roughly calculated from the peak area of Ar1s and C1s in XPS. The concentrations were corrected for the partial ionization cross section calculated from the model of Scofield (1973) and the inelastic mean free path of electrons (Seah and Dench, 1979). Using the SRIM 2006 code simulation (http://www.srim.org), we calculated that the 3-keV Ar ions bombarding the carbon stopped at a depth of $5.1 \pm 1.6 \mathrm{~nm}$, which is deeper than the inelastic mean free path of Ar1s photoelectrons $(1.3 \mathrm{~nm})$ whose kinetic energy is $294 \mathrm{eV}$. However, the surface concentration of Ar de-

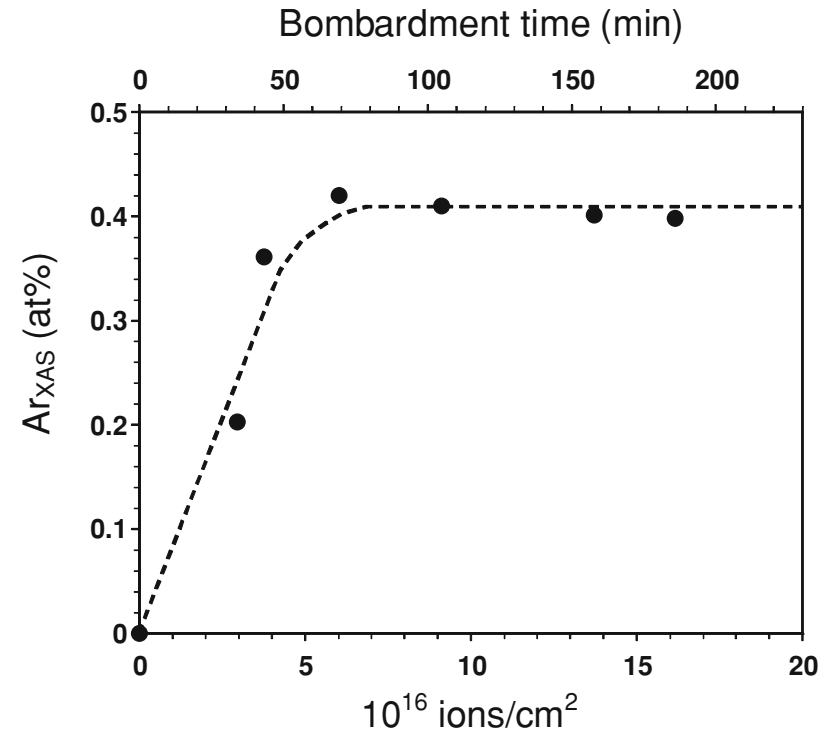

Fig. 3. Argon concentration in carbon nanotube estimated by XAS in stepwise bombardment. ArXAS reaches quasi-saturated value of 0.4 atom\% after a 1 -h bombardment.

termined by the XPS method corresponds to the maximum concentration in the target material because the surface of the target material is continuously eroded by the ion beam and, eventually, implanted Ar comes to have a characteristic distribution. Figure 2 shows the depth profile of the implanted $\mathrm{Ar}$ in $\mathrm{C}, \mathrm{Fe}$, olivine and coal. When the ions have bombarded the target material for a long time and the surface is ablated to a depth of $10 \mathrm{~nm}$ or more, the shape of the depth profile depends only on the energy of the bombarding ions and the composition of the target material, and does not depend on the sputtering rate and trapping efficiency. This graph clearly shows that the Ar concentration reaches its maximum on the surface layer, where it can be detected by the XPS analysis. The concentration of Ar estimated by XPS is, therefore, much higher than that estimated by XAS because XAS yields information on a much deeper part of the samples as described in the following text.

After a long period of $\mathrm{Ar}^{+}$bombardment, the Ar concentration reaches a quasi-saturated concentration, which is determined by two factors: the trapping efficiency of the bombarding Ar ions, and the sputtering rate of the implanted layer of the target material, with the latter being influenced by the depth profile of the implanted Ar (Baba et al., 1992a). Indeed, the Ar concentration reaches saturation in the stepwise irradiation. Figure 3 presents the change in Ar concentration in carbon nanotube with $\mathrm{Ar}^{+}$bombardment. About $6 \times 10^{16}$ ions are required to achieve the quasisaturated concentration. Other target materials also reach the quasi-saturated concentration by the ion implantation method, similar to the case of carbon nanotube.

\section{Results}

\subsection{Ar in carbon allotropes}

The quasi-saturation Ar concentrations in carbon allotropes estimated from XAS ( $\left.\mathrm{Ar}_{\mathrm{XAS}}\right)$ and XPS ( $\left.\mathrm{Ar}_{\mathrm{XPS}}\right)$ are summarized in Table 3 . The large difference between $\mathrm{Ar}_{\mathrm{XAS}}$ and $\mathrm{Ar}_{\mathrm{XPS}}$ is due to the difference in the detection 
Table 3. Quasi-saturated Ar concentrations.

\begin{tabular}{ccccccc}
\hline \multirow{2}{*}{ Target } & \multicolumn{2}{c}{$\operatorname{Ar}_{\mathrm{XAS}}{ }^{\mathrm{a}}$} & & \multicolumn{2}{c}{$\operatorname{Ar}_{\mathrm{XPS}}(\text { atom } \%)^{\mathrm{b}}$} \\
\cline { 2 - 3 } \cline { 5 - 6 } $\mathrm{mol} / \mathrm{mol}$ & & & atom $\%$ & $\mathrm{~mol} / \mathrm{g}$ \\
\hline Fullerene & 0.41 & $3.4 \times 10^{-4}$ & & 6.8 & $4.9 \times 10^{-3}$ \\
Carbon nanotube & 0.40 & $3.3 \times 10^{-4}$ & & 6.9 & $5.0 \times 10^{-3}$ \\
HOPG & 0.79 & $6.5 \times 10^{-4}$ & & 12.0 & $7.8 \times 10^{-3}$ \\
CVD Diamond & 0.74 & $6.1 \times 10^{-4}$ & & 11.5 & \multicolumn{2}{c}{$7.6 \times 10^{-3}$} \\
Olivine & 0.72 & $3.6 \times 10^{-4}$ & & \multicolumn{2}{c}{ n.d. $^{\mathrm{c}}$} \\
Fe & 3.70 & $6.7 \times 10^{-4}$ & & 25.3 & \multicolumn{2}{c}{$4.9 \times 10^{-3}$} \\
\hline
\end{tabular}

uncertainties are lower than $13 \%$

${ }^{\mathrm{b}}$ uncertainties are lower than $17 \%$

${ }^{c}$ not determined

depth of each analysis. The $\mathrm{Ar}_{\mathrm{XPS}}$ of the four carbon allotropes ranges from 6.8 to 12.0 atom\%, indicating that $\mathrm{Ar}$ ions are efficiently implanted and retained in the carbon materials. The concentrations observed in this experiment can be considered to correspond to the targets' retentivity of Ar if their sputtering rates are similar. Since all of the carbon allotropes are composed entirely of carbon, their sputtering yields are indeed comparable. Therefore, the maximum surface concentrations of Ar can be used as a convenient index of Ar retentivity.

Of the four allotropes, HOPG has the highest Ar concentration, up to $12.0 \%$. This result may be due to the trapping site of Ar: Ar atoms are presumably retained between the aromatic carbon layers (Hahn and Kang, 2000), and HOPG is composed entirely of aromatic carbon layers.

In addition to HOPG, CVD diamond has a high Ar concentration, the highest being $11.5 \%$, which is consistent with the fact that diamond in primitive meteorites is a main phase for primordial noble gases, HL, P3, and P6 (Huss and Lewis, 1994a, b, 1995). Although fullerene and carbon nanotube have lower concentrations than diamond and HOPG, the Ar concentrations of carbon allotropes are considerably higher than those of diamond and graphite implanted by other processes, such as shock implantation $\left(0.25 \mathrm{~cm}^{3}\right.$ STP/g atm Ar in diamond; Matsuda and Nagao, 1989) and vapor-growth diamond and graphite synthesized in ambient noble gas $\left(<0.15 \mathrm{~cm}^{3} \mathrm{STP} / \mathrm{g}\right.$ atm $\mathrm{Ar}$ in graphite; Fukunaga et al., 1987; 2-7 $\mathrm{cm}^{3} \mathrm{STP} / \mathrm{g}$ atm Ar in diamond; Matsuda et al., 1991). The ion bombardment is one of the most efficient methods of implanting the noble gas into target materials.

Diamonds are found to have a high retentivity of $\mathrm{Ar}$ in our study, although the trapping site of Ar is clearly different from that of the other three allotropes, since diamond is composed entirely of $\mathrm{sp}^{3}$ covalent bonds. In order to compare the trapping situations of implanted Ar, we plotted the XAS spectra near the Ar $K$-edge (Fig. 4). Figure 4 shows that there is no remarkable difference among the five spectra, with the $K$-edge absorption peaks appearing at the same photon energy. Although noble gas is an inert element and, presumably, does not bond to carbon, it is known that the $K$-edge absorption spectra of noble gases are affected by the internal pressure (intrinsic stress) of amorphous carbon (Lacerda et al., 2002). In the case of Xe implanted in transition metals, the trapping efficiency and the extraatomic relaxation energy correlate with the number of $d-$ electrons in the metals, indicating that the trapped atoms

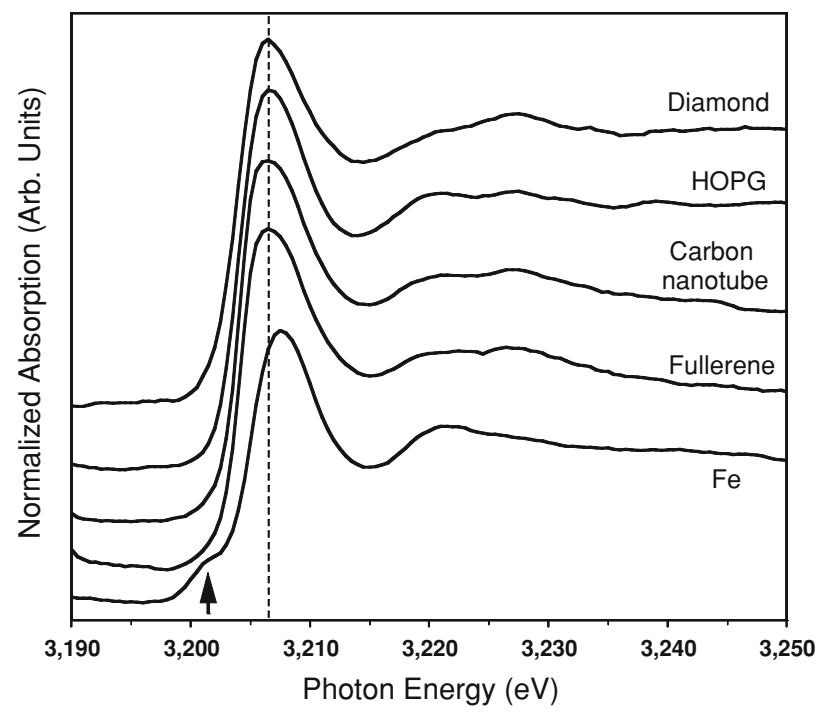

Fig. 4. X-ray absorption spectra for four carbon allotropes and Fe near the Ar $K$-edge. The carbon allotropes have similar spectra, and $K$-edge absorptions appear at the same position of approximately $3207 \mathrm{eV}$ (dotted line). An arrow indicates the small peak of Fe appearing at $3202 \mathrm{eV}$.

feel the surrounding metal potential (Baba et al., 1992a, b). However, we cannot find any such difference in the XAS shown in Fig. 4, indicating that the trapping states of implanted Ar in the carbon allotropes are similar to each other or, alternatively, that the difference in the trapping states is undetectable in this analysis. On the other hand, the XAS of Ar-bombarded Fe presented in the graph clearly differs from the spectra of the carbon allotropes; the $K$-edge absorption that shifts to a higher energy and the appearance of a smaller peak at $3202 \mathrm{eV}$ demonstrates that XAS is sensitive to the surrounding potential. The small absorption may be assigned to the splitting of $1 \mathrm{~s} 4 \mathrm{p}$ resonance, indicating some kind of the interaction between $\mathrm{Ar}$ and $\mathrm{Fe}$ atoms and suggesting that the trapping state of $\mathrm{Ar}$ in Fe differs from that in carbon allotropes.

\subsection{Estimation of Ar concentration from XAS}

When the surface concentration of Ar is low $(<1 \%$ atom\%), the Ar concentration cannot be estimated from the peak area of XPS due to the low sensitivity of XPS. In addition, XPS can only detect Ar atoms existing in the 1-nmdeep surface layer. In order to estimate the total amount of implanted Ar, we have developed a convenient method for estimating of Ar content using the Ar $K$-edge jump ratio in XAS. The edge-jump ratio is defined as $I_{\text {on }} / I_{\text {off }}$ where $I_{\text {on }}$ and $I_{\text {off }}$ represent the intensities of the resonant and nonresonant X-ray absorption, respectively, which are calculated from the two regression lines shown in Fig. 5(a). The two lines are calculated from the method of least squares using the Victoreen formula for the range of 3100-3195 and $3250-3300 \mathrm{eV}$ for $I_{\text {off }}$ and $I_{\mathrm{on}}$, respectively. Relative Ar content can roughly be estimated from the magnitude of the edge jump of the XAS appearing at $3200 \mathrm{eV}$. The XAS is simply composed of the combination of the spectra of Ar and the matrix elements. In the case of the carbon allotropes, the matrix element is only carbon. The Ar content can, therefore, be qualitatively estimated from the edge-jump ratio. 

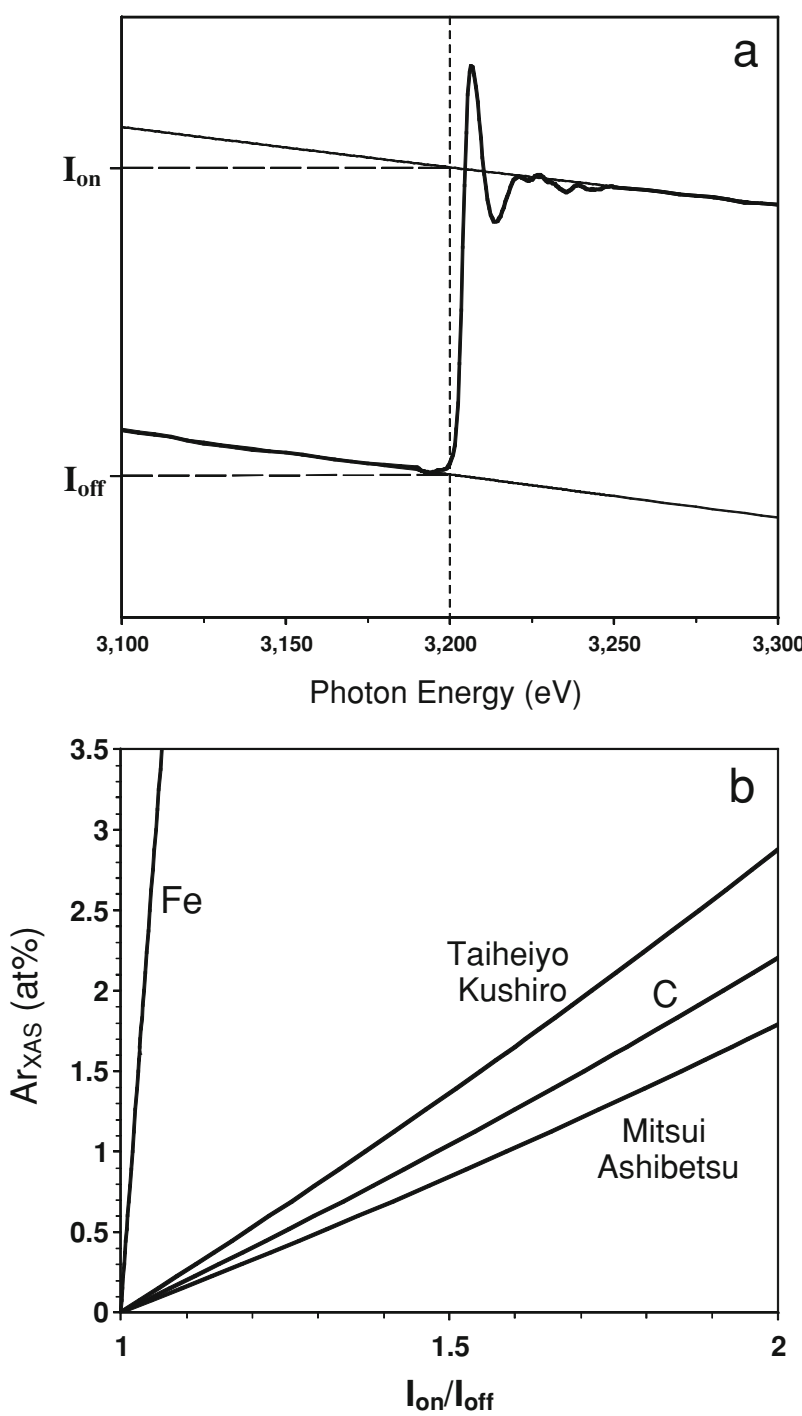

Fig. 5. $I_{\text {on }}$ and $I_{\text {off }}$ of Ar $K$-edge appeared at approximately $3200 \mathrm{eV}$ in XAS (a) and a diagram of edge-jump ratio $\left(I_{\text {on }} / I_{\text {off }}\right)$ versus estimated $\mathrm{Ar}$ concentration in carbon, iron, and two coals (b). Ar XAS can be estimated by the edge-jump ratio using the total photon cross section, which is calculated from the elemental composition of target material and photo cross sections for each element.

This ratio correlates with the Ar concentration, so the concentration can be used as an index of Ar retentivity. The relationship between $I_{\text {on }} / I_{\text {off }}$ and the Ar concentration $\left(\mathrm{Ar}_{\mathrm{XAS}}\right)$ in carbon, iron, and two coals is shown in Fig. 5(b). Since the correlation line depends on the elemental composition of the target material, the function of each line is calculated from the integration of the total photon cross section of all target elements (Berger et al., 2005). Although this estimate is only semiquantitative, it is sufficiently accurate to compare the concentrations of Ar implanted in the target materials. The concentration estimated by the method is systematically lower than the concentration calculated by the XPS method because XAS can detect atoms that exist in deeper parts of the target materials than XPS. Table 3 shows that the Ar concentration estimated by the XAS method is much lower than that estimated by XPS, indicating that the concentrations reflect the fact that Ar exists within a depth of a few tens of nanometers.
$\mathrm{Ar}_{\mathrm{XAS}}$ for San Carlos olivine and $\mathrm{Fe}$ are shown in Table 3. Olivine has 0.72 atom\% Ar, which is comparable with diamond and HOPG, and Fe has a very high Ar concentration of 3.70 atom\%. Although these values should not be simply compared with the value for carbon allotropes described above, it appears that the former can also be good Ar carriers, particularly Fe.

\subsection{Ar concentration of kerogens}

Figure 6 shows a comparison of the edge jumps in XAS for HOPG, fullerene, and a kerogen sample (Nukibetsu coal). The edge jump of the kerogen is significantly lower than those of the carbon allotropes, although the peak position of the $K$-edge absorption appears at the same position (approx. $3207 \mathrm{eV}$ ). This very low jump ratio indicates a low retentivity of Ar in kerogen. All other kerogen samples measured in our study have edge-jump ratios comparable to that of Nukibetsu coal, although these samples vary greatly in terms of their elemental composition (Fig. 1). Argon concentrations estimated by the XAS method are summarized in Table 4. It is very surprising that the Ar retentivity of kerogen is much lower than that of the carbon allotropes: the average Ar retentivity of all the coals tested in our study is only $0.04-0.08$ atom\%. Since there is no significant difference between the concentrations of samples with and without pyridine treatment, the existence of bitumen can be considered not to influence Ar retentivity at all. There is also no significant correlation between $\operatorname{Ar}_{X A S}$ and chemical composition, maturity, HI, OI, and PI. This result presumably indicates that these terrestrial kerogens do not have ordered structures to capture Ar.

The unexpectedly low Ar concentrations are not due to artificial reasons, such as electrical conductivity and the condition of the sample. We confirmed that there are no significant differences in the shape of XAS and in the estimated Ar concentration of the powder and chip samples

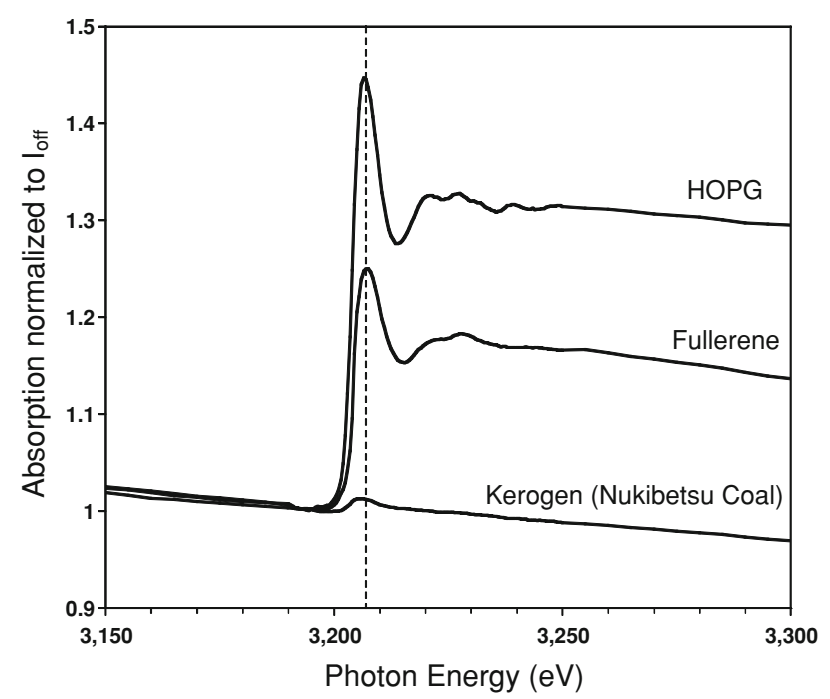

Fig. 6. Edge jumps of HOPG, fullerene, and Nukibetsu coal. The ordinate is normalized to $I_{\text {off }}$. Dotted line shows the peak position of Ar $K$-edge appearing at $3207 \mathrm{eV}$. The edge jump of Nukibetsu is extremely small compared with the carbon allotropes, showing a low Ar concentration. All other coals have comparable edge jump with Nukibetsu coal not shown. 
Table 4. Quasi-saturated Ar concentrations of coals.

\begin{tabular}{|c|c|c|}
\hline \multirow{2}{*}{ Target } & \multicolumn{2}{|c|}{$\mathrm{Ar}_{\mathrm{XAS}}{ }^{\mathrm{b}}$} \\
\hline & atom $\%$ & $\mathrm{~mol} / \mathrm{g}$ \\
\hline Shin-sarufutsu & 0.06 & $7.6 \times 10^{-5}$ \\
\hline Mitasui Ashibetsu (chip) & 0.07 & $8.9 \times 10^{-5}$ \\
\hline Mitasui Ashibetsu & 0.08 & $1.0 \times 10^{-4}$ \\
\hline Mitsui Bibai & 0.05 & $5.8 \times 10^{-5}$ \\
\hline Mitsui Bibai (py) $^{\mathrm{a}}$ & 0.06 & $6.7 \times 10^{-5}$ \\
\hline Tomiuchi Fukuyama & 0.07 & $5.9 \times 10^{-5}$ \\
\hline Tomiuchi Fukuyama (py) ${ }^{\mathrm{a}}$ & 0.08 & $6.6 \times 10^{-5}$ \\
\hline Nukibetsu (py) ${ }^{\mathrm{a}}$ & 0.05 & $5.0 \times 10^{-5}$ \\
\hline Obihiro & 0.05 & $6.3 \times 10^{-5}$ \\
\hline Taiheiyo Kushiro (chip) & 0.06 & $7.1 \times 10^{-5}$ \\
\hline Taiheiyo Kushiro & 0.07 & $8.3 \times 10^{-5}$ \\
\hline Taiheiyo Kushiro (py) $^{\mathrm{a}}$ & 0.04 & $4.6 \times 10^{-5}$ \\
\hline
\end{tabular}

of Mitsui Ashibetsu and Taiheiyo Kushiro, as presented in Table 4. After carefully consideration, we conclude that the low Ar concentrations of kerogens estimated by XAS certainly reflect actual low concentrations of Ar.

\section{Discussion}

Our experimental results show that terrestrial Type II and III kerogens are not good carriers of Ar compared to other materials due to the former lacking stable trapping sites for Ar. This result appears to be inconsistent with the assumption that Q-noble gases are trapped in kerogen-like materials. Although the estimated Ar concentrations of kerogens may be high enough to explain the concentrations of phase Q-noble gases, we do not know a true concentration of Qnoble gases because the carrier of Q-noble gases is not yet separated, and its concentration may be much higher than that of the acid residue of the primitive meteorite. Phase Q is distributed in the fine-grained rims around chondrules together with presolar diamonds (Nakamura et al., 1999). Q gases may be trapped in very thin coatings of grains, since grain surface catalysis is one of the mechanisms of organic formation (e.g., Ferrante et al., 2000). Therefore, the weight of the carrier phase of $\mathrm{Q}$ where the noble gases are actually preserved is very little compared to the entire weight of the acid residue. If the size of the Q-containing grains is of the same order as that of the nano diamonds (about $2.6 \mathrm{~nm}$ in diameter) or the coatings of grains are very thin, the Ar concentration of kerogens (0.04-0.08 atom\%) may not be able to account for the concentration of Q-noble gases.

If the carrier phase of Q-noble gases is really carbonaceous material, two hypothetical situations can explain the results found in this work. In the first explanation, extraterrestrial carbonaceous materials are not similar to the terrestrial kerogen tested in our study, although the structural difference cannot be investigated by current analytical methods. If the structure of phase $\mathrm{Q}$ is quite different from that of terrestrial kerogen despite their similar chemical composition, the trapping efficiency of Ar may also be different. In the second explanation, phase $\mathrm{Q}$ is very similar to terrestrial kerogen, but Ar was encapsulated into the phase by some chemical and/or physical process in the early solar nebula or protosolar molecular cloud, a process which was not reproduced in our study. However, in our experiments, the condition of ion bombardment is mild and only the retentivity of $\mathrm{Ar}$ is considered. The kerogens tested do not seem to be suitable as the material of phase Q because their retentivity of Ar is relatively low. When kerogens are extremely fragile and more easily sputtered by Ar irradiation than carbon allotropes, the apparent retentivity of Ar will become small. However, it cannot be said that kerogens are extremely fragile compared to carbon allotropes, since the structures of both these materials are formed by carbon bonds, and their bond energies are comparable or the same.

The amount of Ar remaining in the target material is controlled by four factors: density of the ion current, bombardment duration, sputtering rate, and trapping efficiency (escape rate). Since the bombardment of $\mathrm{Ar}^{+}$was conducted under the same conditions for all samples and only the quasi-saturated concentration is discussed here, the density of the ion current and bombardment duration cannot be the reason for the low concentration of Ar in the kerogens. The sputtering rate of kerogen is presumably comparable with that of the carbon allotropes for the reason described above. Thus, the remaining factor, the trapping efficiency, is the main reason. If Ar atoms are in a thermodynamically stable state in the target material, the trapping efficiency rises naturally when thermal diffusion is overcome. If Ar has no such stable state, or in other words, if there is no suitable trapping site, bombarded Ar can escape to the outside easily. Energetic Ar ions are decelerated and retained in the carbon network of kerogen but they are not fixed to the stopped place due to the lack of a suitable trapping site. The temporarily stopped atoms eventually are driven outside by thermal diffusion. The fact that carbon allotropes can preserve much of the Ar from the 3-keV Ar${ }^{+}$beam indicates that these materials can continue to retain Ar and that they are not notably destroyed by the ion incidence. Although Type II and III kerogens contain a higher concentration of aromatic carbon than Type I, their structure is disordered compared with crystalline material, appearing like a jumble of aromatic, cyclic, and aliphatic carbons (Behar and Vandenbroucke, 1987). This structure presumably is one of the reasons it obstructs the fixation of Ar.

Based on this discussion, we may conclude that the latter explanation (where phase Q is similar to terrestrial kerogen) is not likely, although it cannot be completely dismissed, and that the more acceptable explanation is that phase $Q$ is not similar to terrestrial kerogen. In support of the former explanation, the structure of phase $\mathrm{Q}$ may have a higher degree of order than the terrestrial kerogen tested in our study because the greatest difference between kerogen and carbon allotropes is the degree of order of the molecular structure. Kerogens have a larger variety of carbon bonding than carbon allotropes.

For the latter explanation to be the case, a very improbable condition is required: that only the kerogen-like carbonaceous material traps noble gas, and all other materials do not. Although presolar grains evidently existed as solid matters in the early solar nebula, there are no Q-noble gases in known presolar grains. The kerogen-like carbonaceous 
material was, therefore, spatially separated from the presolar grains in the trapping process of Q-noble gases. Moreover, a special physical/chemical process may be needed to tightly fix heavy noble gases in this material. If various materials were exposed to ambient noble gas at the same time and if noble gases were injected into them by some processes, many materials in addition to kerogen-like matter must have high concentrations of noble gases and the extremely biased distribution of noble gases in phase Q would not be seen. Although aqueous alteration in the meteorite parent body can remove noble gases from silicate phases, the process cannot remove Q-noble gases from presolar diamond and graphite because they are stable even in $\mathrm{HCl} / \mathrm{HF}$.

Our results show that all materials other than kerogens have a higher Ar retentivity than kerogens. It is thus very difficult to concentrate heavy noble gases in low-retentivity material only.

Although the trapping efficiency of Ar probably correlates to the release temperature of the implanted Ar, we were unable to verify this correlation in our experiments. Stepwise heating experiments for kerogen and carbon allotropes should be conducted in future studies. A strong indication that kerogens cannot be analogous to phase $\mathrm{Q}$ would be if the release temperature of implanted Ar in kerogens were to be lower than $1000^{\circ} \mathrm{C}$.

\section{Conclusion}

Argon concentrations in carbon allotropes and kerogens after Ar ion bombardment were estimated from XPS and XAS recorded using synchrotron X-rays. The concentrations were used as indexes of the relative retentivity of Ar. Our results show that Type II and III kerogens clearly have much a lower Ar retentivity than the four carbon allotropes (HOPG, CVD diamond, carbon nanotube, and fullerene), olivine, and Fe. This unexpected result suggests that the terrestrial kerogens are not suitable as the carrier phase of Q-Ar.

Since phase $\mathrm{Q}$ in reality retains an extremely large amount of heavy noble gases and the gases are tightly bound to the phase, the phase Q-forming material must be an excellent noble gas carrier. Our study, however, shows that the terrestrial kerogens are inferior to other carbon materials in terms of noble gas retentivity. This result implies that phase Q may not be similar to terrestrial kerogen, especially in the coal samples tested in our experiments. We suggest that the phase Q may not resemble kerogen and that its structure is more ordered than that of kerogen. It is unlikely that the structure of phase Q is similar to terrestrial kerogen.

Acknowledgments. Yves Marrocchi and an anonymous referee are thanked for their constructive comments and suggestions. This study was conducted under the approval of Photon Factory Program Advisory Committee (Contract No. PF-PAC 2006G309) and was supported by a Grant-in-aid for Scientific Research (19740337) from JSPS. We thank F. Esaka for his meaningful advices.

\section{References}

Anders, E., Noble gases in meteorites: evidence for presolar matter and superheavy elements, Proc. R. Soc. A, 374, 207-238, 1981.

Baba, Y., H. Yamamoto, and T. A. Sasaki, Trapping of low-energy xenon ions in surfaces of transition metals, Nucl. Instr. Methods. B, 63, 391-
394, 1992a.

Baba, Y., H. Yamamoto, and T. A. Sasaki, XPS and XAES measurements on trapped rare gases in transition metals, Nucl. Instr. Method. B, 66 , 424-432, 1992b.

Behar, F. and M. Vandenbroucke, Chemical modeling of kerogen, Org. Geochem., 11, 15-24, 1987.

Berger, M. J., J. H. Hubbell, S. M. Seltzer, J. Chang, J. S. Coursey, R. Sukumar, and D. S. Zucker, XCOM: Photon Cross Sections Database, Web Version 1.3, available at http://physics.nist.gov/xcom, 2005.

Busemann, H. and O. Eugster, The trapped noble gas component in achondrites, Meteorit. Planet. Sci., 37, 1865-1891, 2002.

Busemann, H., H. Baur, and R. Wieler, Primordial noble gases in "phase Q" in carbonaceous and ordinary chondrites studied by closed-system etching, Meteorit. Planet. Sci., 35, 949-973, 2000.

Durand, B. and J. C. Monin, Kerogen, edited by B. Durand, 113 pp., Éditions Technip, Paris, 1980.

Espitalié, J., M. Madec, and B. Tissot, Role of mineral matrix in kerogen pyrolysis: influence on petroleum generation and migration, Bull. Am. Assoc. Pet. Geol., 64, 59-66, 1980.

Fan, X., C. Liu, T. Yu, Y. Shi, J. Li, M. Ye, H. Guo, N. Xiao, Y. Pen, and F. Cai, Preparation and ion implantation of solid $\mathrm{C}_{60}$ films, Surf. Coat. Tech., 65, 219-223, 1994.

Ferrante, R. F., M. H. Moore, J. A. Nuth III, and T. Smith, Laboratory studies of catalysis of $\mathrm{CO}$ to organics on grain analogs, Icarus, 145, 297-300, 2000.

Frick, U., Noble gas fractionation during synthesis of carbonaceous matter, Proc. Lunar Planet. Sci. Conf., 1961-1973, 1979.

Fukunaga, K., J. Matsuda, K. Nagao, M. Miyamoto, and K. Ito, Noble-gas enrichment in vapour-growth diamonds and the origin of diamonds in ureilites, Nature, 328, 141-143, 1987.

Gardinier, A., S. Derenne, F. Robert, F. Behar, C. Largeau, and J. Maquet, Solid state CP/MAS 13C NMR of the insoluble organic matter of the Orgueil and Murchinson meteorites: Quantitative study, Earth Planet. Sci. Lett., 184, 9-21, 2000.

Greene, J. P., J. Nemanich, G. E. Thomas, and S. L. Schiel, Noble gas sputtering calculations using TRIM, Nucl. Instr. Methods. A, 397, 9198, 1997.

Hahn, J. R. and H. Kang, Spatial distribution of defects generated by hyperthermal $\mathrm{Ar}^{+}$impact onto graphite, Surface Sci., 446, L77-L82, 2000.

Hartman-Stroup, C., The effect of organic matter type and organic carbon content on Rock-Eval hydrogen index in oil shales and source rocks, Org. Geochem., 13, 1051-1060, 1987.

Hayatsu, R., R. G. Scott, and R. E. Winane, Comparative structural study of meteoritic polymer with terrestrial geopolymers coal and kerogen, Meteoritics, 18, 310, 1983.

Hirao, N., Y. Baba, T. Sekiguchi, and I. Shimoyama, Reconstruction of Xray photoelectron spectroscopy system installed at the synchrotron soft X-ray beamline, JAEA-Tech, 039, 2006.

Hohenberg, C. M., N. Thonnard, and A. Meshik, Active capture and anomalous adsorption: new mechanisms for the incorporation of heavy noble gases, Meteorit. Planet. Sci., 37, 257-267, 2002.

Huss, G. R. and R. S. Lewis, Noble gases in presolar diamonds I: Three distinct components and their implications for diamond origins, Meteoritics, 29, 791-810, 1994a.

Huss, G. R. and R. S. Lewis, Noble gases in presolar diamonds II: Component abundances reflect thermal processing, Meteoritics, 29, 811-829, $1994 b$.

Huss, G. R. and R. S. Lewis, Presolar diamond, SiC, and graphite in primitive chondrites: Abundances as a function of meteorite class and petrologic type, Geochim. Cosmochim. Acta, 59, 115-160, 1995.

Huss, G. R., R. S. Lewis, and S. Hemkin, The "normal planetary" noble gas component in primitive chondrites: Compositions, carrier and metamorphic history, Geochim. Cosmochim. Acta, 60, 3311-3340, 1996.

Kastner, J., H. Kuzmany, L. Palmetshofer, P. Bauer, and G. Stingeder, Doping of fullerenes by ion implantation, Nucl. Instr. Methods Phys. Res. B, 80-81, 1456-1459, 1993.

Lacerda, R. G., L. R. Tessler, M. C. dos Santos, P. Hammer, F. Alvarez, and F. C. Marques, EXAFS study of noble gases implanted in highly stressed amorphous carbon films, J. Non-Crystalline Solids, 299-302, 805-809, 2002.

Lewis, R. S., B. Srinivasan, and E. Anders, Host phase of a strange xenon component in Allende, Science, 190, 1251-1262, 1975.

Marrocchi, Y., S. Derenne, B. Marty, and F. Robert, Interlayer trapping of noble gases in insoluble organic matter of primitive meteorites, Earth Planet. Sci. Lett., 236, 569-578, 2005a. 
Marrocchi, Y., A. Razafitianamaharavo, L. J. Michot, and B. Marty, Low pressure adsorption of $\mathrm{Ar}, \mathrm{Kr}$ and $\mathrm{Xe}$ on carbonaceous materials (kerogen and carbon blacks), ferrihydrite and montmorillonite: implications for the trapping of noble gases onto meteoritic matter, Geochim. Cosmochim. Acta, 69, 2419-2430, 2005 b.

Matsuda, J. and K. Nagao, Noble gas emplacement in shock-produced diamonds, Geochim. Cosmochim. Acta, 53, 1117-1121, 1989.

Matsuda, J. and T. Yoshida, The plasma model for the origin of the phase Q: an experimental approach and the comparison with the labyrinth model, Meteorit. Planet. Sci., 36, A127, 2001.

Matsuda, J., K. Fukunaga, and K. Ito, Noble gas studies in vapor-growth diamonds: Comparison with shock-produced diamonds and the origin of diamonds in ureilites, Geochim. Cosmochim. Acta, 55, 2011-2023, 1991.

Murae, T., FT-IR spectroscopic studies of major organic matter in carbonaceous chondrites using microscopic technique and comparison with terrestrial kerogen, Proc. NIPR Symp. Antarct. Meteorites, 7, 262-274, 1994.

Nakamura, T., K. Nagao, and N. Takaoka, Microdistribution of primordial noble gases in CM chondrites determined by in situ laser microprobe analysis: Decipherment of nebular processes, Geochim. Cosmochim. Acta, 63, 241-255, 1999.

Ott, U., R. Mack, and S. Chang, Noble-gas-rich separates from the Allende meteorite, Geochim. Cosmochim. Acta, 45, 1751-1788, 1981.

Ozima, M., R. Wieler, B. Marty, and F. A. Podosek, Comparative studies of solar, Q-gases and terrestrial noble gases, and implications on the evolution of the solar nebula, Geochim. Cosmochim. Acta, 62, 301-314, 1998.

Remusat, L., S. Derenne, and F. Robert, Conventional and TMAH assisted pyrolysis on the insoluble organic matter of Orgueil and Murchison (abstract \#1230), 34th Lunar and Planetary Science Conference, CDROM, 2003.
Seah, M. P. and W. A. Dench, Quantitative electron spectroscopy of surfaces: a standard data base for electron inelastic mean free paths in solids, Surf. Interface Anal., 1, 2-11, 1979.

Scofield, J. H., Theoretical photoionization cross sections from 1 to 1500 $\mathrm{keV}$, Lawrence Livermore Laboratory, University of California, Livermore California, USA, 1973.

van Krevelen, D. W., Graphical-statistical method for the study of structure and reaction processes of coal, Fuel, 29, 269-284, 1950.

Wacker, J. F., Laboratory simulation of meteoritic noble gases: III. Sorption of neon, argon, krypton and xenon on carbon. Elemental fractionation, Geochim. Cosmochim. Acta, 53, 1421-1433, 1989.

Wacker, J. F., M. G. Zadnik, and E. Anders, Laboratory simulation of meteoritic noble gases: I. Sorption of xenon on carbon: trapping experiments, Geochim. Cosmochim. Acta, 49, 1035-1048, 1985.

Watanabe, S., N. S. Ishioka, T. Sekine, A. Osa, M. Koizumi, H. Shimomura, K. Yoshikawa, and H. Muramatsu, Production of endohedral ${ }^{133}$ Xe-fullerene by ion implantation, J. Radioanal. Nucl. Chem., 255, 495-498, 2003a.

Watanabe, S., N. S. Ishioka, H. Shimomura, H. Muramatsu, and T. Sekine, Dose dependence of the production yield of endohedral ${ }^{133} \mathrm{Xe}$-fullerene by ion implantation, Nucl. Instr. Methods Phys. Res. B, 206, 399-402, $2003 b$.

Wieler, R., E. Anders, H. Baur, R. S. Lewis, and P. Signer, Noble gases in 'phase Q': Closed-system etching of an Allende residue, Geochim. Cosmochim. Acta, 55, 1709-1722, 1991.

Zadnik, M. G., J. F. Wacker, and R. S. Lewis, Laboratory simulation of meteoritic noble gases: II. Sorption of xenon on carbon: etching and heating experiments, Geochim. Cosmochim. Acta, 49, 1049-1059, 1985.

T. Osawa (e-mail: osawa.takahito@jaea.go.jp), N. Hirao, N. Takeda, and Y. Baba 